\title{
Primary Non-Hodgkin's Lymphoma involving Parotid Gland: A Rare Entity
}

\author{
${ }^{1}$ Sushrut Vaidya, ${ }^{2}$ Srivalli Natrajan, ${ }^{3}$ Sneha Kadam
}

\begin{abstract}
Non-Hodgkin's lymphomas constitute 25 to $40 \%$ of all lymphomas. The gastrointestinal tract is the most common site followed by the head and neck area. Salivary gland involvement is a very rare entity and is involved in 0.5 to $5 \%$ of all cases. Among all the major salivary glands, the parotid gland is commonly involved followed by the submandibular glands. Misdiagnosis often leads to unnecessary diagnostic procedures, which leads to delay in initiation of appropriate treatment. It is often difficult to diagnose the distinction between lymphoma developing primarily in the parotid gland tissue and in the intraparotid lymph nodes. As per the reports, primary tumors of the parotid gland show no characteristic features on diagnostic imaging, reflecting none of their histological findings. We represent a case of a 61 -year-old female who presented with painless, gradually increasing mass in the right parotid region. Imaging studies were suggestive of an infiltrative mass lesion involving right the parotid gland. The patient underwent superficial parotidectomy along with the excision of the part of the deep lobe posterior to submandibular gland with facial nerve preservation. Histopathology of the resected specimen shows infiltrates of lymphocytes into the glandular parenchyma destroying its normal lobular organization and disrupting the normal glandular architecture.
\end{abstract}

Keywords: Non-Hodgkin's lymphoma, Parotid gland, Salivary gland.

How to cite this article: Vaidya S, Natrajan S, Kadam S. Primary Non-Hodgkin's Lymphoma involving Parotid Gland: A Rare Entity. J Contemp Dent 2017;7(1):71-75.

\section{Source of support: Nil}

Conflict of interest: None

\section{INTRODUCTION}

Lymphomas appear most commonly in lymph nodes and parenchymal organs, such as liver and spleen, while other localizations are less frequent. They are classified as Hodgkin and non-Hodgkin types, and these two are divided into subtypes according to cell morphology and

\footnotetext{
${ }^{1}$ Professor, ${ }^{2}$ Professor and Head, ${ }^{3}$ Postgraduate Student

${ }^{1-3}$ Department of Oral and Maxillofacial Surgery, Mahatma Gandhi Mission's Dental College and Hospital, Navi Mumbai Maharashtra, India

Corresponding Author: Sneha Kadam, Postgraduate Student, Department of Oral and Maxillofacial Surgery Mahatma Gandhi Mission's Dental College and Hospital, Navi Mumbai, Maharashtra, India, Phone: +918108496035, e-mail: snehakadam.id@gmail.com
}

the characteristics of concurrent elements. ${ }^{1}$ Parotid gland lymphomas are a rare primary tumor, although $70 \%$ of all salivary gland lymphomas appear in the parotid gland. ${ }^{2}$ They originate from intraparotid or periparotid lymph nodes, or from lymphoid elements of other pathological states in the gland, such as sialadenitis, cysts with the presence of lymphoid tissue, parenchymal neoplasms with the lymphoid component present, and autoimmune illnesses, especially Sjögren's syndrome. Over the past 10 years, many of the researchers have agreed that the primary localization of lymphomas in parotid salivary gland is very rare, although in recent decades more frequent than earlier. $^{3}$

The lymphoma is defined as primary when there is no detectable disease outside the salivary gland. A small subset of head and neck non-Hodgkin's lymphoma (NHL) is a primary parotid gland lymphoma. The overall incidence is about $0.5 \%$ of all tumors, 3 to $5 \%$ of salivary gland neoplasm, and 5\% of extranodal lymphomas. In contrast to other extranodal locations of NHL, parotid gland involvement is more likely to be of low grade and the patients have a better prognosis than those with other extranodal NHLs. ${ }^{4}$ The most common subtypes of NHL present in the salivary glands are extranodal marginal zone B-cell lymphoma of the mucosa-associated lymphoid tissue (MALT) type, follicular B-cell lymphoma, and diffuse large B-cell lymphoma.

Parotid NHL being an uncommon neoplasm comprises MALT and non-MALT lymphomas. Both types of lymphoma vary in prognosis and treatment. Preoperatively, primary NHL in the salivary glands is not anticipated by most of the surgeons. It is difficult to give a definitive report based on either frozen section or fine-needle aspiration biopsy by the pathologist also. With such a difficulty in pre- and intraoperative diagnosis, it usually ends up in unnecessary radical operations to the patient. Various literature shows that primary tumors of the parotid gland show no characteristic features in diagnostic imaging, reflecting none of their histological findings. ${ }^{5}$ Therefore, it is difficult to differentiate benign conditions from malignant tumors, and most patients are subjected to surgical procedures before a definitive diagnosis has been made. In the present case report, we describe a case of NHL of the parotid gland. 


\section{CASE REPORT}

A 61-year-old female presented with right-sided parotid swelling since 6 months. Initially it was small in size, which gradually increased to size measuring approximately $5 \times 4 \mathrm{~cm}$. On examination, the swelling was nontender, fixed to the underlined structure, firm-to-hard in consistency, and present in a preauricular region extending from helix of the ear superiorly and inferiorly toward the ear lobule. The skin over the swelling has no change in coloration as compared with surrounding skin (Fig. 1). The facial nerve examination was found to be normal. There was no other significant family or personal history. No other abnormality was found in the oral cavity, nasopharynx, larynx, or the ears. There was no palpable enlargement of cervical lymph nodes. There was no clinical evidence of Sjögren's syndrome, lymphadenopathy, recurrent gland swelling, or unexplained xerostomia. Differential diagnosis of pleomorphic adenoma, Warthin's tumor, and parotid lymphoma was made. Although rarely observed in the parotid gland, lymphoma should also be considered in the differential diagnosis. The patient was staged according to the Ann Arbor staging system (Table 1). Staging consisted of a general physical examination,

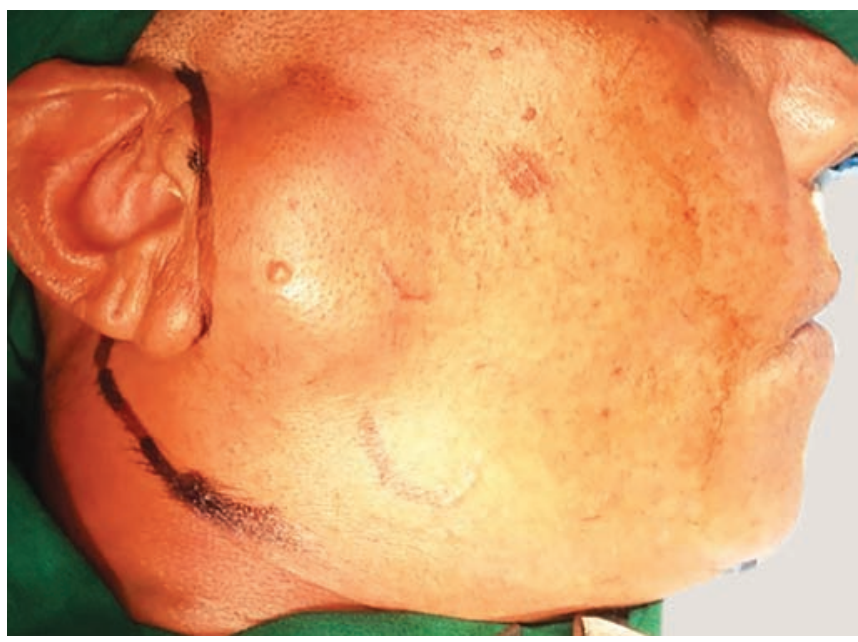

Fig. 1: Preparation of surgical site by preauricular incision

Table 1: Ann Arbor staging system for NHL

\begin{tabular}{|c|c|}
\hline Stage & Description \\
\hline 1 & $\begin{array}{l}\text { Involvement of a single lymph node region or of a } \\
\text { single extranodal organ or site (I E) }\end{array}$ \\
\hline II & $\begin{array}{l}\text { Involvement of two or more lymph node regions on the } \\
\text { same side of the diaphragm, or localized involvement } \\
\text { of an extranodal site or organ (II E) and one or more } \\
\text { lymph node regions on the same side of the diaphragm }\end{array}$ \\
\hline III & $\begin{array}{l}\text { Involvement of lymph node regions on both sides of } \\
\text { the diaphragm, which may also be accompanied by } \\
\text { localized involvement of an extranodal organ or site (III } \\
\text { E) of spleen (III S) or both (III SE) }\end{array}$ \\
\hline IV & $\begin{array}{l}\text { Diffuse or disseminated involvement of one or more } \\
\text { distant extranodal sites }\end{array}$ \\
\hline
\end{tabular}

Table 2: Stage grouping relevant to treatment planning for $\mathrm{NHL}$

\begin{tabular}{ll}
\hline Limited stage & Advanced stage \\
\hline Ann Arbor I, II & Ann Arbor III, IVa \\
Bulk less than $10 \mathrm{~cm}$ & Bulk greater than $10 \mathrm{~cm}$ \\
No B symptoms & B symptoms \\
\hline
\end{tabular}

hematological screening, computed tomography (CT) of chest, abdomen, and pelvic regions, and bone marrow biopsy. The CT chest and ultrasonography of abdomen and pelvis were normal. In contrast to the complexity of classification, the staging of NHL is straightforward. The patients can be divided into two groups based on Ann Arbor stage: Bulk or size of tumor and presence of B symptoms (Table 2). The B symptoms refer to systemic symptoms of fever, night sweats, and weight loss, which can be associated with both Hodgkin's lymphoma and NHL. The presence or absence of B symptoms has prognostic significance and is reflected in the staging of these lymphomas. The CT scan of head and neck shows solid homogeneous mass with ill-defined border involving right parotid gland, largest in the superficial lobe and extending into the deep lobe (Figs 2A and B). As per the staging system, our patient was under stage I, bulk less than $10 \mathrm{~cm}$, and no B symptoms were observed.

A superficial parotidectomy with part of the deep lobe posterior to submandibular gland was excised with preauricular skin incision. Facial nerve and its terminal branches were preserved with careful dissection. The retromandibular vein was ligated and a globular mass seen arising from the superficial lobe of the parotid gland (Figs 3 and 4). The tumor was removed along with other smaller mass from the deep lobe posterior to submandibular gland (Fig. 5). A corrugated rubber drain was placed for 48 hours (Fig. 6).

\section{Hematoxylin and Eosin-stained Sections}

\section{Under Low Magnification}

Both capsulated and uncapsulated areas of salivary gland tissue and lymph nodes were seen. Infiltrates of lymphocytes are seen infiltrating into the glandular parenchyma destroying its normal lobular organization. Foci of oncocytes were also seen (Fig. 7).

\section{Under Higher Magnification}

Lymphomatous infiltrates that have disrupted the normal glandular architecture were seen. Lymphomatous area shows both monotypic and pleomorphic cells. The presence of numerous large cells with eosinophilic cytoplasm showing monolobed or bilobed nucleus with nuclei can be appreciated in between lymphocytes. Acini adjacent to the lymphoid infiltrate are destroyed (Fig. 8). Many 


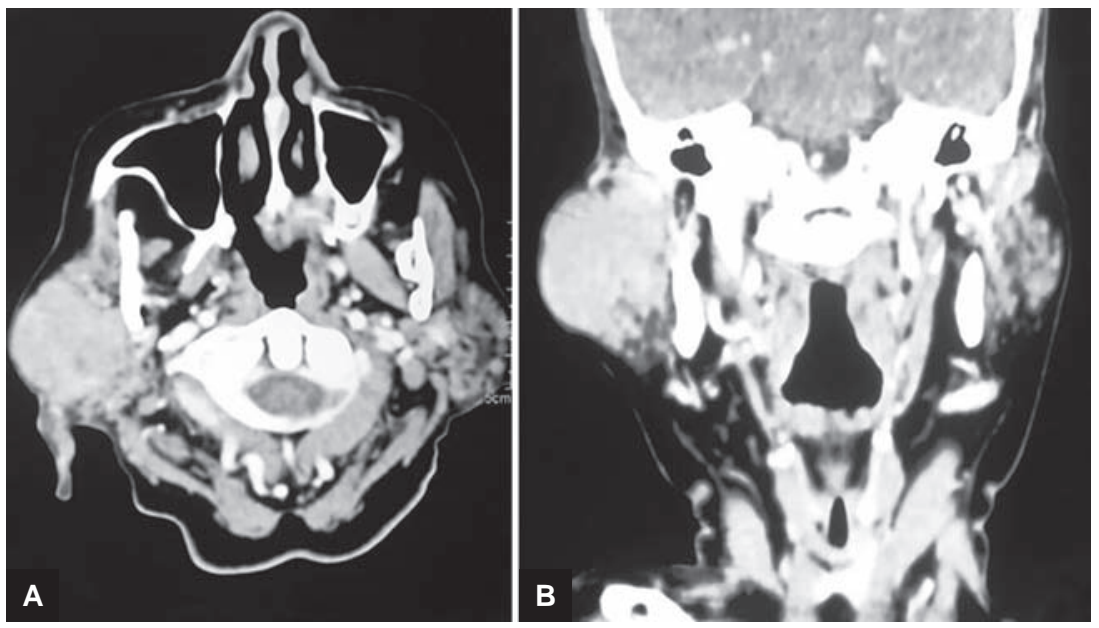

Figs 2A and B: (A) The CT image reflects diffuse homogeneous neoplasm with an illdefined margin occupied in the right parotid gland; and (B) Coronal CT image reflects the solid mass with well-circumscribed margins in the right parotid gland

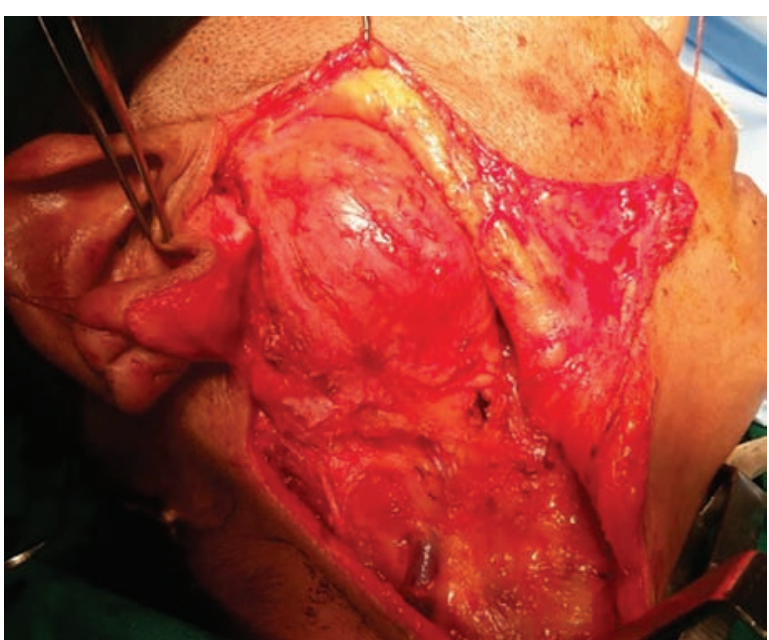

Fig. 3: Intraoperative image reflecting tumor involving parotid superficial lobe

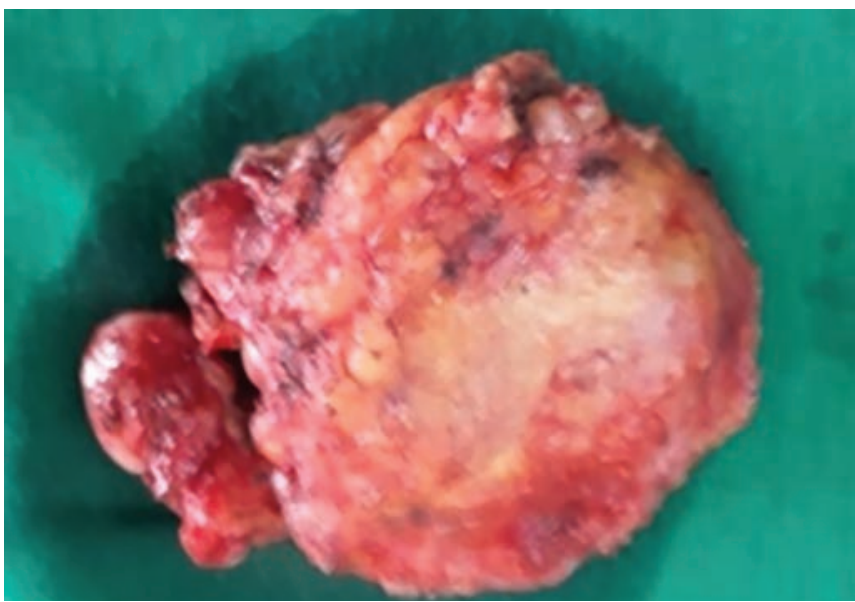

Fig. 5: Resected specimen

scattered salivary gland ducts and epimyoepithelial islands are also seen.

Postoperative recovery was uneventful and facial nerve function showed mild weakness, which improved

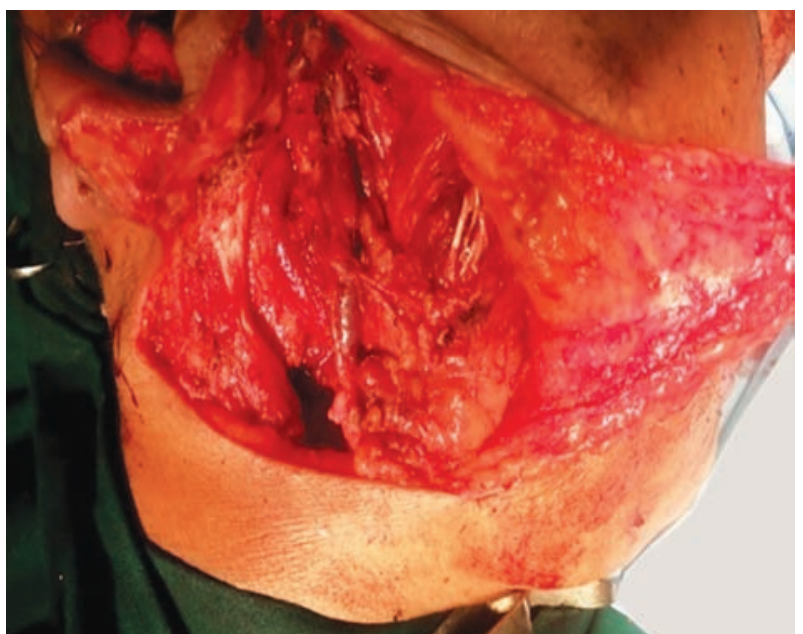

Fig. 4: Postoperative image after removal of tumor

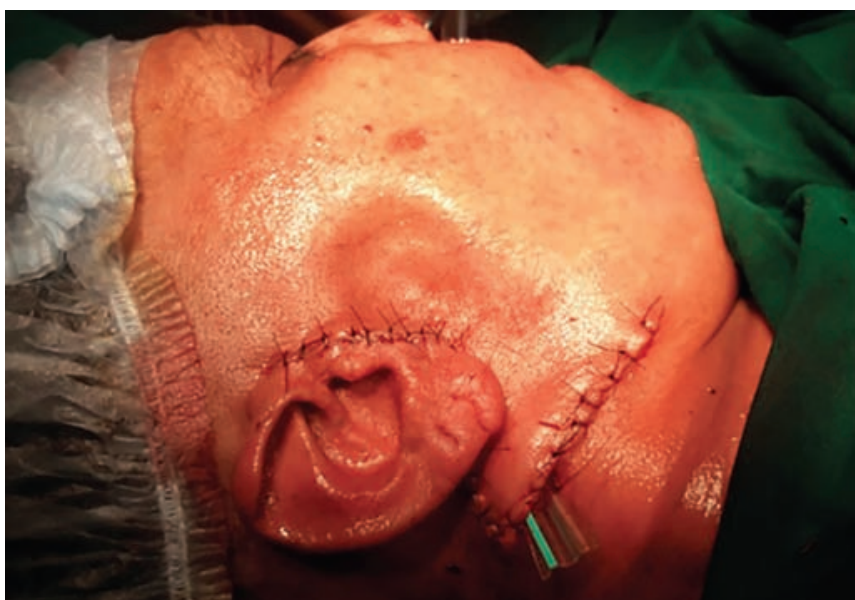

Fig. 6: Postoperative picture of the patient with corrugated rubber drain

by physiotherapy (Fig. 9). The CT scan of the head, neck, chest, and abdomen at 2 and 6 months after the surgery revealed no evidence of lymphoma infiltration. Our patient had isolated surgical treatment without chemotherapy or 


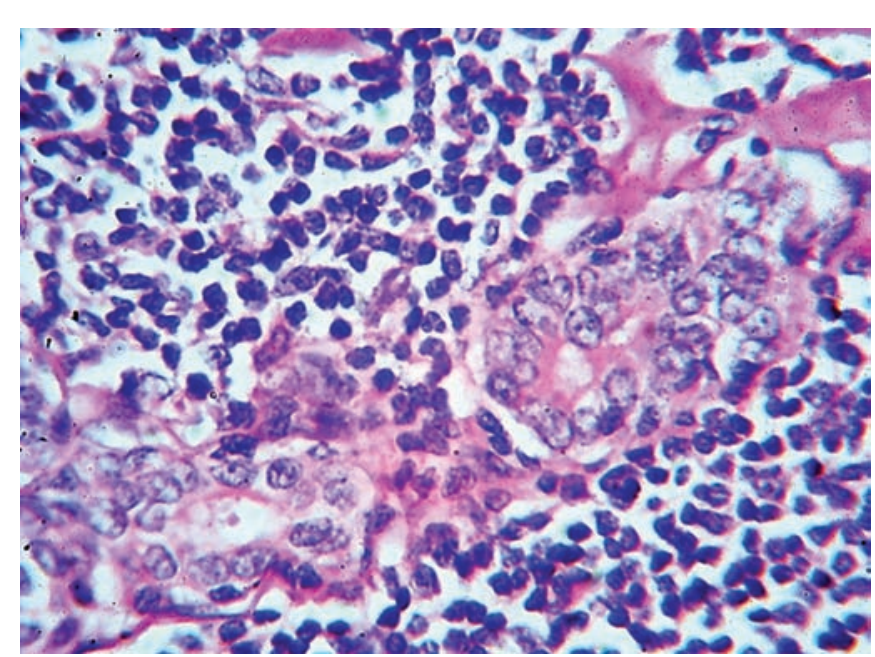

Fig. 7: Histopathological examination of specimen - infiltration of salivary and parenchyma with atypical lymphoid cells

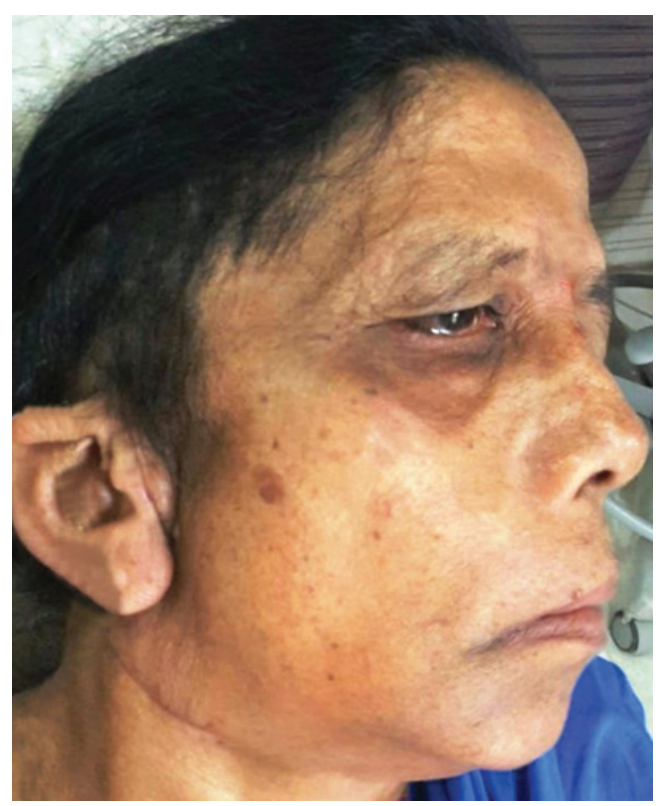

Fig. 9: Seven months postoperative view

radiotherapy. By the time this report was completed, the patient had been followed for 7 months without evidence of recurrence.

\section{DISCUSSION}

Malignant lymphoma of the parotid region may arise from an intraparotid lymph node or in the gland itself. In the intraparotid lymph node, the histological features and natural history of the disease are those of nodal lymphoma in general. Non-Hodgkin's lymphoma of the parotid gland is usually a painless, progressive enlarging mass. ${ }^{6}$ As only about $5 \%$ of all salivary gland tumors are malignant lymphomas, primary salivary gland lymphoma is rarely suspected before biopsy or surgical removal. ${ }^{7}$

Primary salivary gland lymphoma is defined as enlargement of parotid gland at first presentation,

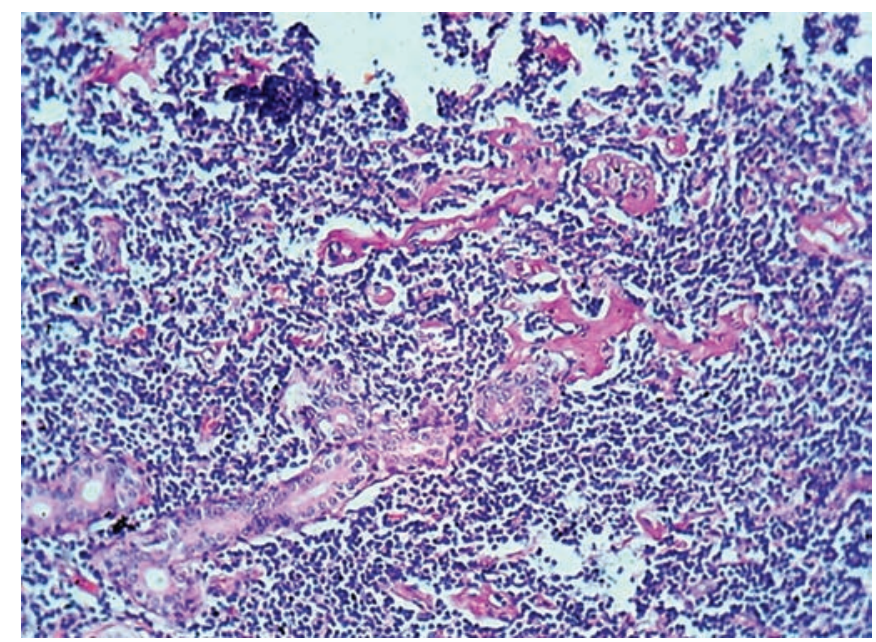

Fig. 8: Dense lymphoid infiltrate with partial obliteration of acini

followed by pathological diagnosis that includes parenchymal involvement of the salivary gland without evidence of lymph node origin..$^{89}$ The majority of salivary gland lymphomas are NHLs of B-cell type, especially MALT lymphomas. The MALT lymphoma is usually associated with chronic inflammatory process, which is either because of an immune process or infection, such as myoepithelial sialadenitis and Sjögren's syndrome. ${ }^{10}$ Schusterman et $\mathrm{al}^{5}$ noticed a progressive increase in the incidence of salivary gland lymphomas during the last several decades. This may result from a combination of factors: (1) referral pattern, (2) increased lifespan of individual, and (3) use of sophisticated molecular techniques. Malignant lymphomas of the parotid gland are uncommon in patients younger than 50 years, with 2:1 male to female ratio. The impact of the disease stage, histological grade, and presence or absence of an underlying autoimmune disease on clinical outcome is controversial.

Primary malignant lymphomas of the parotid gland are uncommon. Clinically, they manifest as a unilateral, slow-growing painless mass. Most of these tumors are B-cell type of NHL. Malignant lymphoma originating in the parotid gland is relatively rare and occurs in 1 to $5 \%$ of tumors, where the parotid gland is the original site of the tumor. ${ }^{9}$ It is believed that the differentiation of malignant lymphoma originating in the parotid gland from other tumors (including benign tumors) is difficult; however, some authors as per the literature have reported that malignant lymphomas showed tumor homogeneity surrounded by well-defined margins and were rarely associated with necrosis. ${ }^{10}$ So as per the observations of the present case, it is indicated that it is necessary to consider the possibility of malignant lymphoma even when the lesion is rapidly increasing in size and exhibits necrosis or an infiltrative tendency in the imaging presentations, strongly suggesting epithelial tumors. Malignant lymphoma originating in 
the parotid gland is histologically described as low-grade NHL that frequently belongs to the B-cell type and rarely relapses into other sites. ${ }^{10} \mathrm{So}$, surgery as prognostic management can be used.

\section{CONCLUSION}

Swelling and lymphomas affecting parotid gland are clinically indistinguishable from other benign or malignant lesions. Imaging modalities and fine-needle aspiration cytology are not always helpful. Hence, the majority of patients require a parotidectomy for definitive diagnosis.

\section{REFERENCES}

1. Cho G, Suh IS, Tak KS, Park YK, Ko EY, Sung HM, Shin MK. Primary parotid non-Hodgkin's lymphoma: case report. J Korean Cleft Palate-Craniofac Assoc 2010 Oct;11(2):99-102.

2. Gleson MJ, Bennet MH, Cawson RA. Lymphoma of salivary gland. Cancer 1986 Aug;58(3):699-704.

3. Schmid U, Helboon D, Lennert K. Development of malignant lymphoma in myoepithelial sialadenitis (Sjögren's syndrome). Virchows Arch A Pathol Anat Histol 1982 Mar;395(1):11-43.
4. Barnes L, Myers EN, Prokopakis EP. Primary malignant lymphoma of parotid gland. Arch Otolaryngol Head Neck Surg 1998 May;124(5):573-577.

5. Schusterman MA, Granick MS, Erickson ER, Newton ED, Hanna DC, Bragdon RW. Lymphomas presenting as a salivary gland mass. Head Neck Surg 1988 Jul-Aug;10(6): 411-415.

6. Carbone PP, Kaplan HS, Musshoff K, Smithers DW, Tubiana M. Report of the committee on Hodgkin's disease staging classification. Cancer Res 1971 Nov;31(11):1860-1861.

7. Dunn P. Kuo TT, Shih LY, Lin TL, Wang PN, Kuo MC, Tang TC. Primary salivary gland lymphoma: a clinico-pathologic study of 23 cases in Taiwan. Acta Haematol 2004 Nov;112(4):203-208.

8. Hyman GA, Wolff M. Malignant lymphoma of the salivary gland. Review of literature and report of 33 new cases, including four cases associated with lymphoepithelial lesion. Am J Clin Pathol 1976 Apr;65(4):421-438.

9. Freling NJM, Molenaar WM, Vermey A, Mooyaart EL, Panders AK, Annyas AA, Thijin CJ. Malignant parotid tumors: clinical use of MR imaging and histologic correlation. Radiology 1992 Dec;185(3):691-696.

10. Nagata M, Kumazawa H, Iwai H, Momotani A, Shiraishi S, Yamashita T. Study of malignant lymphoma in the parotid gland region. Nihon Jibiinkouka Gakkai Kaiho 1996 Jun;99(6): 918-925. 